\title{
- Effect of Slump Neural Mobilization vs Mulligan Bent Leg Raise in Subjects with Lumbar Radiculopathy
}

\author{
IJCRR \\ Section: Healthcare \\ ISI Impact Factor \\ (2020-21): 1.899 \\ IC Value (2020): 91.47 \\ $\operatorname{SJIF}(2020)=7.893$ \\ Copyright@IJCRR
}

\section{Agrawal R ${ }^{1}$, Shah $K^{2}$}

'Professor and Principal, M A Rangoonwala College of Physiotherapy, Azam Campus, Camp, Pune-4110o1, India; ${ }^{2}$ Physiotherapist, M A

Rangoonwala College of Physiotherapy, Azam Campus, Shankarsheth Road, Camp, Pune-4110o1, India.

\section{ABSTRACT}

Introduction: Low back pain is a problem worldwide with a lifetime prevalence reported as 34\% by WHO1. Low back pain(LBP) is experienced by at least $80 \%$ of adults, both men and women equally atleast once in their lifetime. 2 Direct nerve mobilization uses sliders, tensioners and nerve mobilization crossing single joint addressing the interface between various surfaces. The Mulligan BLR technique has been described as means of improving range of SLR(Straight leg raise), AKE(active knee extension) and hamstring flexibility in subjects with LBP or referred thigh pain.

Aims: The aim of this study was to compare the effectiveness of both techniques in treating patients with lumbar radiculopathy.

Methodology: After receiving permission from Institutional Ethics committee, sixty subjects were randomly allocated to Mulligan Bent knee raise and Neural tissue Mobilization group. Lumbar stabilization exercises were given to both groups. Intervention was given for 3 days/week for 3 weeks.

Results: Data was analyzed using SPSS VERSION 17.0. The results showed significant difference in all 3 outcome measures ie SLR, Modified Oswestry Disability Index( MODI) score and NPRS scale in intra- group comparison. But inter-group comparison was non-significant for pain score (NPRS scale) and disability (MODI score) but showed significant difference for passive SLR range where the range improved to a greater extent with slump neural mobilization technique than mulligan bent leg raise.

Conclusion: We conclude that both mulligan bent leg raise and slump neural mobilization are equally effective in reducing pain and disability. Also, both the techniques improve range of SLR but slump neural mobilization increases SLR range to a greater extent than mulligan bent leg raise.

Key Words: Mulligan bent leg raise, Slump neural mobilization, Lumbar radiculopathy, Modified ODI, NPRS, SLR

\section{INTRODUCTION}

Low back pain is a problem worldwide with a lifetime prevalence reported as $34 \%$ by WHO. ${ }^{1}$ Low back pain(LBP) is experienced by at least $80 \%$ of adults, both men and women equally atleast once in their lifetime. ${ }^{2}$ It is the most common cause of disability in individuals under 45 years of age and $3 \mathrm{rd}$ most common cause in 45-60 year age group. Various spinal structures such as paravertebral muscles, ligaments, facet joints annulus fibrosus and spinal nerve roots have been suggested as the cause of pain apart from disc herniation and stenosis. It has been suggested that if nociceptive inputs continue overtime it may result in functional, chemical and structural alterations in peripheral and central nervous systems. ${ }^{3}$
The nervous system is a viscoelastic organ. Mechanics and physiology of the nervous system are interdependent and forms the basis for the concept of neurodynamics. Neurodynamic sliding (NDS) integrates both the musculoskeletal and nervous systems to achieve pain reduction or increased ROM in the extremities ${ }^{4}$ Neural mobilization is a set of techniques designed to restore elasticity of nervous system, defined as ability of nerve mechanical interphase to shift in relation to other structures. ${ }^{5}$ The structures which can be moved by these techniques include neuraxis, meninges, nerve roots and peripheral nerves. ${ }^{4}$

Direct nerve mobilization uses sliders, tensioners and nerve mobilization crossing single joint addressing the interface between various surfaces, correcting the posture and ergonomic adjustment. ${ }^{6,7}$ Sliders utilize combinations of joint

\section{Corresponding Author:}

Agrawal R, M A Rangoonwala College of Physiotherapy, Azam Campus, Camp, Pune-411001, India.

Phone: 09922445653; E-mail: ronika1000@rediffmail.com

ISSN: $2231-2196$ (Print)

Received: 07.01 .2021
ISSN: 0975-5241 (Online)

Revised: 23.02 .2021
Accepted: 03.07.2021
Published: 15.02 .2022 
movements to encourage peripheral nerve excursion by increasing elongation at one end of the nerve bed, thereby creating tension from the other end of the nerve. In contrast, tensioners make use of combinations of joint movements that lengthen the nerve bed from both ends, in an effort to elongate the neural connective tissues. ${ }^{8}$

The Mulligan BLR technique has been described as means of improving range of SLR (Straight leg raise), AKE(active knee extension) and hamstring flexibility in subjects with LBP or referred thigh pain. This technique was designed to restore altered activation of hamstring muscle Mulligan Bent Leg Raise technique consists of gentle isometric stretching of hamstring in specific directions in progressively greater positions of hip flexion. It can be tried in patients with gross bilateral limitation of SLR. Mobilization of nervous tissue has been postulated as one of the causes for improvements seen post mulligan BLR. The aim of this technique is to restore normal mobility and decrease low back pain and associated disability. ${ }^{9,10}$

Studies have been conducted to check effectiveness of both Mulligan bent leg raise and slump neural mobilization in LBP and results are found to be significant but very few studies have compared two techniques for its effectiveness in lumbar radiculopathies. The purpose of this study is to compare the effectiveness of both techniques in treating patients with lumbar radiculopathy.

\section{METHODOLOGY}

After receiving permission from Institutional Ethics committee MCES/EC/ZVM/452/18, pre and post-experimental study with purposive sampling was done. Duration of study intervention was 3 weeks. Seventy subjects in the age group of 18 to 60 years, both males and females, having LBP radiating distal to the buttocks with reproduction of symptoms during slump stretching with active knee extension more than 15 degrees in bed side sitting position, subjects with SLR less than 60 degrees with radiation of symptoms were included.

Subjects with symptoms of spinal infection, neoplasm, spinal fractures, positive signs or symptoms suggesting UMN lesion, conditions where SLR is contra-indicated were excluded from the study.

\section{Group A. (Mulligan bent leg raise) $)^{11,12}$}

Subject was in supine lying. Therapist stood on painful side of patient or the side with limited straight leg raise. Therapist placed the patient's flexed knee over his shoulder. Now therapist asked the patient to push the flexed knee in the direction of therapist and then relax. At this point, the therapist pushed his bent knee up as far as possible in the direction of the patient's shoulder on the same side provided there is no pain. If it was painful the patient's leg was rotated medially or laterally. The stretch has to be sustained for several seconds and then the leg is lowered to the bed. After three repetitions marked improvement in straight leg raise was noted. ${ }^{11}$ This was carried in five progressively greater positions of hip flexion.

\section{Group B. (SIump neural mobilization) ${ }^{13}$}

The subject was seated at the edge of bed with knees at 90 degrees of flexion and popliteal surface in contact with the plinth. The patient was asked to slouch the shoulders and thoracic spine while the therapist applied cervical over pressure to ensure consistent pressure, maintaining fully flexed position, and the patient was asked to actively extend the knee. 30 sliders i.e neck flexion with ankle plantarflexion and neck extension with ankle dorsiflexion were performed by the patient actively. For both groups, the treatment was provided thrice weekly for 3 weeks resulting in 9 total treatment sessions.

\section{Lumbar spine stabilization exercises: ${ }^{14}$}

Lumbar spine stabilization exercises were common to both groups under supervision for core muscle strengthening as weakness of core muscle plays a significant role in LBP. These exercises were given following Mulligan's bent leg raise and slump neural mobilization in the form of following with 5 seconds hold and 10 repetitions 1 set per day. Exercises included were

Abdominal draw in, Abdominal draw in with bridging, Quadruped with abdominal draw in, Quadruped alternate arm and leg lift with abdominal draw in, Quadripud with arm lift, Quadripud with leg lift. All exercises were carried out under supervision.

\section{RESULTS}

Comparison of age and sex in bent leg raise group and slump mobilization group was not statistically significant. Hence, they are comparable. Comparison of VAS, SLR and Modified MODI score in Neural Tissue Mobilization and mulligan bent leg raise group showed significant reduction in pain and thus the VAS score reduced consistently from baseline to 3 weeks.

\section{DISCUSSION}

The present study was conducted on sample size of 70 patients for a period of three weeks comparing Mulligan bent leg raise and Slump neural mobilization in subjects with lumbar radiculopathy. The three outcome measures used 
were numerical pain rating scale for pain intensity, Modified ODI for disability and SLR (Straight leg raise).

There was significant reduction in pain and thus the NPRS score reduced consistently from baseline to 3 weeks in Mulligan bent leg raise. One of the reasons for reduction in pain with mulligan bent leg raise technique is improved mechanics of neural structures. ${ }^{12}$ It facilitates caudal movement of neural structures which improves excursion of lumbosacral nerve roots and sciatic nerve at the level of pelvis (hip is kept in flexed position). Also, there is opening of inter-vertebral foramina and central canal which helps disperse intra-neural oedema, relieving pressure gradients and thus hypoxia and hence the pain. ${ }^{15,16,17}$

Also, that bent leg raise technique may have effect on neurophysiological responses which affects muscle stretch tolerance and increase hamstring flexibility ${ }^{18}$ It is suggested in previous studies that increase in hamstring extensibility offloads pelvis of its excessive posterior rotation and provides lumbar range of motion by providing efficient lumbo-pelvic rhythm thus by improving the biomechanics, there is reduction in pain..$^{19}$ Another mechanism for reduction in pain by bent leg raise technique is altered pain perception. Afferent input from muscles and joints during Mulligan bent leg raise may interfere with nociceptive fibres which is consistent with gate control theory proposed by Melzack and Wall. The afferent input blocks the slow $\mathrm{C}$ fibres which subsequently inhibits individuals perception of pain. Alternatively, changes in pain responsiveness may be psychologically mediated, sometimes giving a placebo effect on pain reduction..$^{12,20}$

Table 1 shows intra-group comparison of NPRS score in slump neural mobilization group where patient was given neural sliders. The NPRS score showed significant reduction $(p<0.001)$ in pain over a period of 3 weeks. Neural sliders have hypoalgesic effect on pain pressure threshold. This is supported by a study conducted by Beltran- Alacreu who found neural sliders superior in reducing pain when compared to neural tensioners and placebo.21 Sliders are effective in reducing pain as there is combination of movements in which there is elongation of the nerve at one joint and is simultaneously counterbalanced by reduction in length of the nerve bed at adjacent joint. (Shacklock 2005, Scrimshaw and Maher 2001) ${ }^{22}$. Cleland et al., Gladson et al. mentioned in their study that when nerve root was compressed and microcirculation compromised, the pressure received increases oedema and demyelination. Sliders help to relieve hypoxia and thus associated symptoms by decreasing edema and demyelination because oscillatory movements helps in dispersing oedema and increase axoplasmic flow. Also it directly alleviates neurogenic inflammation. It is hypothesized that mechanosensitivity can be alleviated when nerve movement is within pain- free variations (sliders) which help to reduce nerve compression, tension and friction. ${ }^{23}$
Table 2 shows comparison of mean NPRS score difference in Mulligan bent leg raise and slump neural mobilization group. It shows no one technique proved to be superior over other for reducing pain as there is no statistically significant difference between both the groups $(\mathrm{p}>0.05)$.

When effect on SLR were seen in MBR group as in Table 3 it is seen that there is considerable improvement in range of SLR from baseline to 3 weeks. The p-value is extremely significant $(p=<0.001)$ in both the groups. The increase in range of SLR post BLR may be attributed to decrease in pain and due to post- isometric relaxation of hamstring. Also it helps to release adhesions in the sciatic nerve at the hip level as it passes through gluteus maximus and adductor magnus ( as knees are kept in flexed position). Mobility of sciatic nerve increases in relation to the surrounding structures (especially muscles) without the nerve getting stretched or trapped. ${ }^{9,24}$

Mulligan bent leg raise moves the painful, sensitized nerve tissue similar to 'slider effect' described by Butler (1991) and Elvey and Hall (1997). Another beneficial effect is increased hamstring extensibility due to change in stretch tolerance. Isometric contraction of connective tissue occurs with mulligan bent leg raise and improvement in SLR range can be attributed to that effect. ${ }^{12}$ This is consistent with results of other study where $70 \%$ of improvement in range was due to change in hip flexion.

Table 3 shows intra- group comparison of SLR range of motion in slump neural mobilization group and shows consistent and significant improvement in SLR range from baseline to third week.

There is increased tissue mobility following neurodynamic technique as after removal of pressure by oscillatory sliders the neuron recovers well. Blood circulation and axonal transport are necessary for the functional and structural integrity of neuron. Adverse neural tension may be one of the factor for low back pain and leg pain. ${ }^{10}$ The nervous system is a viscoelastic organ. Safe viscoelastic effects occur within a few seconds of force application to neural structures. To hold a neurodynamic technique longer may place the neural structure at risk due to intraneural ischeimia with time. That is why movement techniques are safer and better tolerated by patients than stretching. ${ }^{4}$

During joint movement the following movements occur in sequence; in the early range the slack is taken up, rapid neural sliding in the mid- range. A slider technique is a large amplitude movement through the mid- range. ${ }^{4}$ Charnley's (1951) and Smith's (1956) classic studies showed that 0-35 degrees, the slack in the sciatic nerve is taken up. The movement is most rapid between 35-70 degrees and ceases between 70-90 degrees.

Table 4 shows comparison of mean difference in SLR and increase in SLR range in both groups (mulligan bent leg raise 
and slump neural mobilization). The results show that both interventions are effective in increasing SLR range of motion but slump neural mobilization increased passive SLR range to a greater extent compared to bent leg raise technique at 3 weeks.

Neural sliders consist of short oscillatory movements sufficient to disperse edema, relieve hypoxia and also reduce associated symptoms. Thus neurodynamic slider techniques work better as an intervention when compared to mulligan bent leg raise. ${ }^{23}$ Dixon and Keating (2000) suggest that improvement in range of SLR must be greater than 6 degrees to state that a real change in range of SLR has occurred. The change in range of SLR does not have immediate benefit with bent leg raise technique and is of clinical relevance only 24 hours after intervention. ${ }^{9}$ In our study we found a considerable improvement in SLR range of 20- 30 degrees with slump sliders and 10- 20 degrees with bent leg raise technique.

Gajendrakumar Patel conducted a study comparing MBLR and slump stretching and found MBLR technique to significantly reduce pain and improve ROM of PSLR than the slump stretching technique. ${ }^{24}$ In contrast, our study found that slump mobilization was more effective in increasing range of SLR and both techniques were equally effective in reducing pain and disability. This variation might be due to the fact that their study excluded patients with SLR range less than 45 degrees and also they used slump stretching i.e tensioner technique whereas in our study we used slump sliders which have proven effective in increasing SLR range to a greater extent as compared to tensioners.

Table 5 shows intra- group comparison for disability due to lumbar radiculopathy in bent leg raise group and shows considerable reduction in MODI (modified Oswestry disability index) from baseline to 3 weeks. Thus, Mulligan bent leg raise technique is a significant intervention to reduce disability. As pain decreased and SLR range increased, the overall disability in daily activities decreased and thus MODI score improved over 3 weeks. The decrease in pain and increase in hamstring extensibility which offloads the pelvis of the excess posterior rotation and improves lumbo-pelvic rhythm and thus helps in reducing disability by improving biomechanical efficiency. ${ }^{12}$

Table 5 shows intra-group comparison for disability due to lumbar radiculopathy in slump group and shows significant reduction in disability by decreasing MODI score from baseline over 3 weeks. The improvement in functional disability by slump neural sliders may be due to increase in sliding effects which improves mobility of nerves in relation to surrounding structures. ${ }^{23}$ Also, reduction in pain by alleviating neurogenic inflammation, reducing hypoxia and decreasing mechanosensitivity makes the patient more functional. Overall, reduction in pain is one of the main reason for reducing disability in patients with radiculopathy.
Table 6 shows the comparison of MODI score in bent leg raise technique and slump mobilization group. Both techniques are equally effective in decreasing disability due to lumbar radiculopathy and no one technique proved to be superior than other. Decrease in pain is one of the major factors for reduction in disability in both the groups. Also lumbar stabilization exercises may have helped in reducing disability by activation of core muscles. ${ }^{2}$

Lumbar spine stabilization exercises were kept in common for both groups. One set of 10 repetitions with 5 second hold was given for all patients under supervision. Constant loading and unloading of spine leads to increased wear and tear and degenerative changes and hence the pain. ${ }^{25}$

Subjects with LBP have shown both asymmetry and atrophy of the multifidus muscle on the side of pain, and a decreased ability to recruit multifidus in patients with chronic LBP and deficits in the ability to recruit the transversus abdominis across all sub-categories of LBP. ${ }^{2}$ Malik et al. showed that combination of lumbar stabilization exercises with SLR mobilization or slump mobilization is superior to stabilization exercises alone for improving SLR range of motion in patients with LBP. ${ }^{26}$ These findings suggest that anyone with LBP may benefit from the inclusion of stabilization exercises.

\section{CONCLUSION}

We conclude that Mulligan bent leg raise and slump neural mobilization are equally effective in reducing pain, disability and improving range of SLR in patients with Lumbar Radiculopathy but slump neural mobilization increases SLR range to a greater extent than Mulligan bent leg raise.

\section{ACKNOWLEDGEMENT}

We thank whole heartedly all the subjects for participating in our study. Authors acknowledge the immense help received from the scholars whose articles are cited and included in references of this manuscript. The authors are also grateful to authors / editors / publishers of all those articles, journals and books from where the literature for this article has been reviewed and discussed.

\section{Financial Assistance: Nil}

\section{Conflict of Interest: Nil}

\section{REFERENCES}

1. Wilson E, Payton O, Donegan-Shoaf L, Dec K. Muscle energy technique in patients with acute low back pain: a pilot clinical trial. Journal of Orthopaedic \& sports Physical therapy. 2003 Sep;33(9):502-12. 
2. Nagrale AV, Patil SP, Gandhi RA, Learman K. Effect of slump stretching versus lumbar mobilization with exercise in subjects with non-radicular low back pain: a randomized clinical trial. Journal of Manual \& Manipulative Therapy. 2012 Feb $1 ; 20(1): 35-42$.

3. Nee RJ, Butler D. Management of peripheral neuropathic pain: integrating neurobiology, neurodynamics, and clinical evidence. Physical Therapy in sport. 2006 Feb 1;7(1):36-49.

4. Shacklock M. Neurodynamics. Physiotherapy. 1995 Jan 1;81(1):9-16.

5. Modic MT, Obuchowski NA, Ross JS, Brant-Zawadzki MN, Grooff PN, Mazanec DJ et al. Acute low back pain and radiculopathy: MR imaging findings and their prognostic role and effect on outcome. Radiology. 2005 Nov; 237(2):597-604.

6. Devillé WL, van der Windt DA, Dzaferagic A, Bezemer PD, Bouter LM. The test of Lasegue: systematic review of the accuracy in diagnosing herniated discs. Spine. 2000 May 1;25(9):1140-7.

7. Meszaros TF, Olson R, Kulig K, Creighton D, Czarnecki E. Effect of $10 \%, 30 \%$, and $60 \%$ body weight traction on the straight leg raise test of symptomatic patients with low back pain. Journal of Orthopaedic \& Sports Physical Therapy. 2000 Oct;30(10):595-601.

8. Stankovic R, Johnell O, Maly P, Wilmer S. Use of lumbar extension, slump test, physical and neurological examination in the evaluation of patients with suspected herniated nucleurs pulposus. A prospective clinical study. Manual therapy. 1999 Feb 1;4(1):25-32.

9. Hall T, Hardt S, Schäfer A, Wallin L. Mulligan bent leg raise technique - a preliminary randomized trial of immediate effects after a single intervention. Manual therapy. 2006 May $1 ; 11(2): 130-5$.

10. Phadnis TR, Bhave SM. Comparative. Study of Mulligan Bent Leg Raise vs. Hold Relax ProprioceptiveNeuromuscularFacilitationonHamstringTightnessinHighSchoolStudent.JournalofPhysiotherapy \& Physical Rehabilitation.2018;03(01).

11. Mulligan BR. Manual therapy. NAGS, SNAGS, MWM etc. 1999:120.

12. Babu VK, Akalwadi A, Kumar SN, Mahendrabhai UM. Immediate effect Of neurodynamic sliding technique versus mulligan bent leg raise technique on hamstring flexibility in asymptomatic individuals. International Journal of Physiotherapy. $2015 \mathrm{Aug}$ 1;2(4):658-66.
13. Lee JH, Kim TH. The treatment effect of hamstring stretching and nerve mobilization for patients with radicular lower back pain. Journal of physical therapy science. 2017;29(9):1578-82.

14. Kisner C, Colby LA, Borstad J. Therapeutic exercise: foundations and techniques. Fa Davis; 2017 Oct 18.

15. Kmiecik J, Frattini C, DiNicola A, Wallace S, Cooper K. ART vs. Graston and Their Effects on Hamstring Flexibility. September 2011. Accessed February. 2012.

16. Panjabi MM, Takata KO, Goel VK. Kinematics of lumbar intervertebral foramen. Spine. 1983;8(4):348-57.

17. Louis R. Vertebral radicular and vertebromedullary dynamics. Anatomiaclinica 1981;3:1-11

18. Harvey LA, Byak AJ, Ostrovskaya M, Glinsky J, Katte L, Randomised trial of the effects of four weeks of daily stretch on extensibility of hamstring muscles in people with spinal cord injuries. Australian Journal of Physiotherapy. 2003 Jan 1;49(3):176-81.

19. Phansopkar PA, Kage V. Efficacy of mulligan's two leg rotation and bent leg raise techniques in hamstring flexibility in subjects with acute non-specific low back pain: randomized clinical trial. Int J Physiother Res 2014;2:733-41

20. Melzack R, Wall PD. Pain mechanisms: a new theory. Science. 1965 Nov 19;150(3699):971-9.

21. Beltran-Alacreu H, Jiménez-Sanz L, Carnero JF, La Touche R. Comparison of hypoalgesic effects of neural stretching vs neural gliding: a randomized controlled trial. Journal of Manipulative and Physiological Therapeutics. 2015 Nov 1;38(9):644-52.

22. Shacklock M. Improving application of neurodynamic (neural tension) testing and treatments: a message to researchers and clinicians. Manual therapy. 2005;3(10):175-9.

23. Adel SM. Efficacy of neural mobilization in treatment of low back dysfunctions. Journal of American Science.2011;7(4):566-73.

24. Patel G. To Compare the effectiveness of Mulligan Bent Leg Raising and Slump Stretching in Patient with Low Back Pain. Indian Journal of Physiotherapy and Occupational Therapy - An International Journal.2014;8(3):24.

25. Agrawal R, Panjwani N. Prevalence of Musculoskeletal Pain in Male Grocery Store workers. Indian Journal of Public Health Research \& Development. 2015;6(1):70-5.

26. Malik N, Kataria C, Sachdev NB. Comparative effectiveness of straight leg raise and slump stretching in subjects with low back pain with adverse neural tension. Int $\mathrm{J}$ Health Rehabil Sci.2012;1(1):2-10.

Table 1: Comparison of NPRS score at baseline, 1wk and 3wks in MBR and NTM group

\begin{tabular}{|c|c|c|c|c|c|c|c|c|}
\hline \multirow[b]{2}{*}{$\begin{array}{l}\text { NPRS } \\
\text { score at }\end{array}$} & \multicolumn{4}{|c|}{ MBR } & \multicolumn{4}{|c|}{ NTM } \\
\hline & Meanı +SD & Mean $2+$ SD & t value & P value & Meanı + SD & Mean 2+ SD & t Value & P Value \\
\hline Baseline\& 1wk & $6.28+1.61$ & $4.25+1.62$ & 4.76 & $<0.0001$ & $6.21+1.9$ & $3.87+1.8$ & 4.87 & $<0.0001$ \\
\hline 1wk \& 3wks & $4.25+1.62$ & $1.97+1.3$ & 4.95 & $<0.0001$ & $3.76+1.8$ & $1.7+1.3$ & 5.03 & $<0.0001$ \\
\hline Baseline \& $3 w \mathrm{ks}$ & $6.28+1.61$ & $1.97+1.3$ & 4.65 & $<0.0001$ & $6.21+1.9$ & $1.7+1.3$ & 4.67 & $<0.0001$ \\
\hline
\end{tabular}

Interpretation: Table shows statistically significant difference when NPRS score is compared for the mulligan bent leg raise and neural tissue mobilization group. Pain reduced significantly from baseline to 1 week and further from 1 week to 3 week with $\mathrm{p}<0.0001$ for both groups. 
Table 2: Comparison of mean NPRS score difference in MBR and Neural Tissue Mobilization group

\begin{tabular}{|c|c|c|c|c|c|c|c|}
\hline \multirow{2}{*}{$\begin{array}{l}\text { NPRS } \\
\text { Mean difference }\end{array}$} & \multicolumn{2}{|c|}{$\operatorname{MBR}(\mathbf{n = 3 2})$} & \multicolumn{2}{|c|}{ Neural Tissue ( $\mathbf{n}=33$ ) } & \multirow{2}{*}{$\begin{array}{c}\text { Z } \\
\text { Value }\end{array}$} & \multirow{2}{*}{$\begin{array}{c}\mathbf{P} \\
\text { Value }\end{array}$} & \\
\hline & Mean & SD & Mean & SD & & & \\
\hline Baseline - 1wk & 2.03 & 1.150 & 2.45 & 1.063 & 1.50 & 0.13 & Not significant \\
\hline $1 w k-3 w k s$ & 2.28 & 1.508 & 1.85 & 1.417 & 1.10 & 0.27 & Not significant \\
\hline Baseline - 3wks & 4.41 & 1.915 & 4.52 & 1.716 & 0.37 & 0.71 & Not significant \\
\hline
\end{tabular}

Interpretation: Table shows comparison of mean NPRS score difference in Mulligan bent leg raise group and Neural Tissue Mobilization group using $\mathrm{Z}$ test. It shows that there is no statistically significant difference at baseline-1 week, 1 week -3 weeks and baseline -3 weeks as $p>0.05$ i.e. both the interventions (Mulligan bent leg raise and Neural Tissue mobilization) were equally effective in reducing the NPRS score.

Table 3: Shows comparison of SLR range in MBR and neural Tissue mobilization group

\begin{tabular}{|c|c|c|c|c|c|c|c|c|}
\hline & MBR & & & & $\begin{array}{l}\text { Neural Tissue } \\
\text { Mobilization }\end{array}$ & & & \\
\hline SLR at & Meanı+ SD & Mean2+SD & T value & Pvalue & Meanı +SD & Mean2+SD & T Value & P Value \\
\hline $\begin{array}{l}\text { Baseline } \\
\text { - 1wk }\end{array}$ & $52.41+8.25$ & $61.66+9.56$ & 18.65 & $<0.0001$ & $53.52+10.3$ & $64.94+9.39$ & 18.70 & $<0.0001$ \\
\hline $1 w k-3 w k s$ & $61.66+9.56$ & $72.16+10.9$ & 20.06 & $<0.0001$ & $64 \cdot 94+9 \cdot 39$ & $80.61+10.6$ & 21.05 & $<0.0001$ \\
\hline $\begin{array}{l}\text { Baseline } \\
-3 w k s\end{array}$ & $52.41+8.25$ & $72.41+10.9$ & $14 \cdot 36$ & $<0.0001$ & $53 \cdot 52+10.31$ & $80.61+10.6$ & 13.23 & $<0.0001$ \\
\hline
\end{tabular}

Interpretation: Table shows statistically significant increase in range of SLR which is consistent from baseline to 1 week to 3 weeks( $\mathrm{p}<0.0001)$.

Table 4: Comparison of mean difference of SLR in MBR and Neural Tissue Mobilization group

\begin{tabular}{lccccccl}
$\begin{array}{l}\text { SLR Mean } \\
\text { difference }\end{array}$ & $\begin{array}{c}\text { MBR(n=32) } \\
\text { Mean }\end{array}$ & SD & $\begin{array}{c}\text { NTM(n=33) } \\
\text { Mean }\end{array}$ & SD & Z Value & & P Value \\
Baseline - 1wk & 9.38 & 2.882 & 11.67 & 3.069 & 3.10 & 0.003 & Significant \\
1wk-3wks & 10.31 & 4.246 & 15.36 & 5.947 & 3.93 & $<0.0001$ & Extremely significant \\
Baseline - 3wks & 19.75 & 5.571 & 27.09 & 7.392 & 4.51 & $<0.0001$ & Extremely significant \\
\hline
\end{tabular}

Interpretation: Table shows comparison of mean SLR score difference in Mulligan bent leg raise group and NTM group using $\mathrm{Z}$ test. It shows that there is statistically significant difference at baseline, 1 week and 3 weeks as $p<0.05$ i.e. NTM is more effective than Mulligan bent leg raise in improving the SLR score.

Table 5: Comparison of MODI score at baseline, iwk and 3wks in MBR group and NTM group

\begin{tabular}{|c|c|c|c|c|c|c|c|c|c|c|c|c|c|}
\hline \multirow[b]{2}{*}{$\begin{array}{l}\text { MODI } \\
\text { score at }\end{array}$} & \multicolumn{6}{|c|}{ MBR } & & \multicolumn{6}{|c|}{ NTM } \\
\hline & Mean & SD & Mean & SD & t Value & P Value & & Mean & SD & Mean & SD & Tvalue & P value \\
\hline $\begin{array}{l}\text { Baseline } \\
\text { - 1wk }\end{array}$ & 20.38 & 6.318 & 16.19 & $5 \cdot 533$ & $4 \cdot 97$ & $<0.0001$ & HS & 19.0 & 6.364 & 14.70 & $5 \cdot 582$ & 5.05 & $<0.0001$ \\
\hline $1 w k-3 w k s$ & 16.19 & $5 \cdot 533$ & 12.13 & 4.950 & 4.95 & $<0.0001$ & HS & 14.7 & $5 \cdot 582$ & 11.39 & $5 \cdot 356$ & 5.03 & $<0.0001$ \\
\hline $\begin{array}{l}\text { Baseline } \\
-3 w k s\end{array}$ & 20.38 & 6.318 & 12.13 & 4.950 & 4.89 & $<0.0001$ & HS & 19.0 & 6.364 & 11.39 & $5 \cdot 356$ & $4 \cdot 9$ & $<0.0001$ \\
\hline
\end{tabular}

Interpretation: TABLE shows statistically significant difference when MODI score is compared for the mulligan bent leg raise and neural tissue mobilization group. MODI score reduced significantly from baseline to 1 week and further from 1 week to 3 week with $\mathrm{P}<0.0001$ for both groups 
Table 6: Comparison of mean MODI score difference in MBR and NTM group

\begin{tabular}{lccccccc} 
MODI score difference & $\begin{array}{c}\text { MBR }(\mathbf{n}=32) \\
\text { Mean }\end{array}$ & SD & $\begin{array}{c}\text { NTM }(\mathbf{n = 3 3}) \\
\text { Mean }\end{array}$ & SD & Z Value & $\begin{array}{c}\mathbf{P} \\
\text { Value }\end{array}$ \\
Baseline \& 1wk & 4.19 & 1.674 & 4.24 & 1.562 & 0.40 & 0.69 & NS \\
1wk \& 3wks & 4.06 & 1.684 & 3.61 & 2.423 & 1.89 & 0.059 \\
Baseline \& 3wks & 8.16 & 2.725 & 7.61 & 2.384 & 1.12 & 0.26 & NS \\
\hline
\end{tabular}

Interpretation: Table shows comparison of mean MODI score difference in Mulligan bent leg raise group and Slump group using Z test. It shows that there is no statistically significant difference at baseline, 1 week and 3 weeks as p>0.o5 i.e. both the interventions (mulligan bent leg raise and neural tissue mobilization) were equally effective in reducing the MODI score. 